\title{
Comparação da evapotranspiração real simulada e observada em uma bacia hidrográfica em condições naturais de cerrado ${ }^{1}$
}

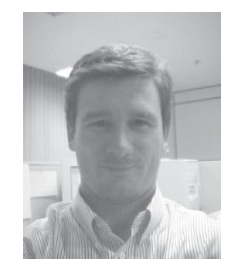

Jorge E.F.W. Lima², Cícero L. da Silva ${ }^{3} \&$ Carlos A. da S. Oliveira ${ }^{4}$

\begin{abstract}
1 Parte da Dissertação apresentada pelo primeiro autor no Curso de Mestrado em Irrigação e Agroambiente da FAV/UnB
2 FAV/UnB. QRSW 04, BI. A1, Ap. 103, CEP 70670-100, Brasília, DF. E-mail: jefwl@conectanet.com.br (Foto)

${ }^{3}$ FAV/UnB. C.P. 04508, CEP 70910-970, Brasília, DF

4 FAV/UnB. C.P. 04508, CEP 70910-970, Brasília, DF
\end{abstract}

Protocolo 117 - 11/09/2000

\begin{abstract}
Resumo: Através deste trabalho, objetivou-se: (i) simular a evapotranspiração real de uma microbacia sob vegetação natural de cerrado, ao longo de um ano, compreendido entre os dias 04/08/98 e 03/08/99; (ii) comparar e analisar o comportamento da evapotranspiração real, do escoamento total e da lâmina de água armazenada no solo, através dos valores medidos e simulados. Utilizando-se a equação do balanço hídrico, determinou-se a evapotranspiração real total do período $(830,53 \mathrm{~mm})$ ao longo do qual foram efetuadas medições intermediárias que permitiram a realização do balanço hídrico em 21 intervalos de tempo. Os valores simulados de evapotranspiração real e de escoamento total ao longo do período analisado não apresentaram boa correlação com os dados obtidos experimentalmente; entretanto, os valores simulados da lâmina atual de água no solo até um metro de profundidade, apresentaram alta correlação com os valores experimentais. Observou-se, no período mais seco do ano, que, apesar da alta evapotranspiração de referência, a evapotranspiração real apresentou-se muito pequena, devido à falta de água disponível para evapotranspirar.
\end{abstract}

Palavras-chave: evapotranspiração, balanço hídrico, Penman-FAO, recursos hídricos

\section{Comparation of simulated and measured data of actual evapotranspiration of a watershed with natural Brazilian savannah conditions}

\begin{abstract}
The main objectives of this research were: (i) to simulate actual evapotranspiration in a small watershed under natural conditions of Brazilian Savannah during a year, between 08/04/98 and 08/03/99; (ii) to compare observed and simulated values of actual evapotranspiration, runoff and water depth stored in the soil. Using the water balance equation, the actual evapotranspiration $(830.53 \mathrm{~mm})$ was computed. During the year, twenty-one intermediate measurements were made in order to use the water balance method for short time intervals. Simulated and measured values of the actual evapotranspiration and the water flow did not have a good correlation. On the other hand, simulated values of the quantity of water stored in the soil to a one meter depth presented results very similar to the measured ones. During the driest period of the year, in spite of the high values of reference evapotranspiration, the actual evapotranspiration presented small values because of the lack of available water to evapotranspirate.
\end{abstract}

Key words: evapotranspiration, water balance, Penman-FAO, water resources

\section{INTRODUÇÃO}

Informações quantitativas de evapotranspiração e evaporação são necessárias nos vários campos científicos que tratam dos numerosos problemas de manejo da água.

Segundo Rebouças et al. (1999) o Distrito Federal é a terceira pior unidade federativa brasileira em disponibilidade hídrica por habitante. Atualmente, conforme estimado por Lima (2000) a disponibilidade hídrica do Distrito Federal, para uma população de cerca de 2.000.000 habitantes (CODEPLAN, IBGE, 2000) é de aproximadamente $1.400 \mathrm{~m}^{3} \mathrm{hab}^{-1} \mathrm{ano}^{-1}$.

De acordo com Beekman (1999) regiões com valores de disponibilidade hídrica anual, per capita, inferiores a $1.700 \mathrm{~m}^{3} \mathrm{hab}^{-1} \mathrm{ano}^{-1}$, representam áreas com risco de falta d'água durante os períodos mais secos do ano. Portanto, o domínio do conhecimento sobre o comportamento dos diferentes parâmetros hidrológicos na região do Distrito Federal é fundamental para minimizar os problemas que a escassez de água pode ocasionar. 
Os métodos diretos de obtenção da evapotranspiração real de determinada área, requerem inúmeras medições; para tanto, são necessários tempo, mão-de-obra, infra-estrutura laboratorial e, conseqüentemente, capital, mas nem sempre é possível contar com todos esses fatores para elaboração de medições.

Segundo Mello et al. (1996) existem entre 50 a 60 equações para a simulação da evapotranspiração. As equações são baseadas em dados meteorológicos para o cálculo da evapotranspiração de referência (ETo). Como constatou Bernardo (1995) alguns dos métodos mais precisos são de difícil aplicação, não só pela complexidade dos cálculos mas, também, por exigirem um grande número de dados meteorológicos, nem sempre disponíveis.

Nas áreas que possuem dados de temperatura, umidade, vento, insolação e radiação, vários autores sugerem o emprego do método de Penman, devido ao fato de apresentar resultados mais precisos para a avaliação dos efeitos do clima sobre a evapotranspiração (Ortolani et al., 1966; Chang, 1968; Jensen, 1973; Silva, 1977; Doorenbos \& Pruitt, 1977; Berlato \& Molion, 1981; Silva, 2000).

Devido às dificuldades na obtenção de dados de parâmetros naturais deve-se, sempre que possível, efetuar a comparação entre dados medidos e simulados, através de modelos matemáticos, como forma de se buscar um método de avaliação que represente, da melhor forma possível, o que ocorre na natureza. Por meio desses modelos pode-se fazer o acompanhamento dos parâmetros naturais de forma mais simples e com custos mais baixos, além das possibilidades de simular panoramas futuros, o que é fundamental, principalmente no que se refere à gestão dos recursos hídricos.

Entre os principais objetivos deste trabalho, estão: (a) a simulação da evapotranspiração real de uma microbacia; (b) a comparação dos dados de evapotranspiração, resultantes da simulação, com valores obtidos por intermédio de um método direto de determinação (método do balanço hídrico) para o mesmo período hidrológico, e (c) a simulação e comparação dos valores de escoamento total e da lâmina de água armazenada no solo, no período de análise.

\section{MATERIAL E MÉTODOS}

\section{Descrição da microbacia}

A área de estudo, microbacia do córrego do Capetinga, está localizada na região central do Brasil, na parte sudeste do Distrito Federal, a 47 $56^{\prime} \mathrm{W}$ de longitude e $15^{\circ} 57^{\prime} \mathrm{S}$ de latitude, conforme pode ser visualizado na Figura 1. A área total é de 963,4 ha e a sua altitude está compreendida entre 1.057 e $1.200 \mathrm{~m}$ acima do nível do mar. As linhas de drenagem têm cerca de $4.330 \mathrm{~m}$ de comprimento, enquanto o curso d'água possui, em média, o comprimento de aproximadamente $2.810 \mathrm{~m}$. A declividade média da bacia é de 1,8\% (Silva \& Oliveira, 1999).

A microbacia do Capetinga fica dentro da Fazenda Água Limpa, de propriedade da Universidade de Brasília e, atualmente, constitui área de preservação ambiental. A vegetação predominante na área é típica do Cerrado brasileiro, sendo constituída por cerrado, campo cerrado, campo limpo e mata ciliar. Através da utilização de ferramenta de geoprocessamento, imagens de satélite, de fotos aéreas e vistorias de campo, foi

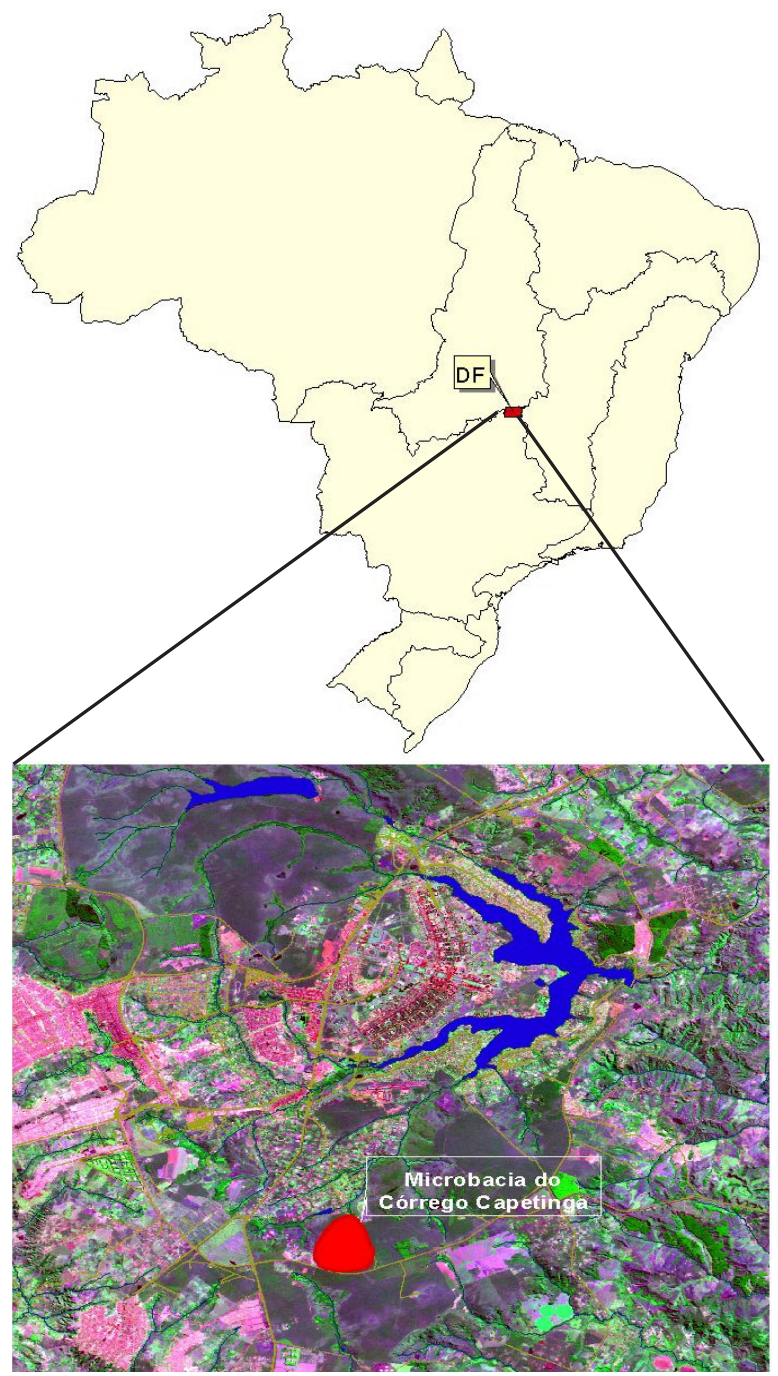

Figura 1. Localização da Bacia do Córrego Capetinga

efetuada a caracterização física da área, assim descrita: (a) cerca de $20 \%$ da área da microbacia são cobertos por vegetação de cerrado sob solo bem estruturado, argiloso, profundo, com subsolo permeável e declividade suave, apresentando boas condições de infiltração da água no solo; (b) 20\% da área são cobertos por campo cerrado, solo argiloso, com média profundidade, moderada permeabilidade, topografia levemente ondulada e boa infiltração; (c) $50 \%$ da área são cobertos por campo limpo sob solo pouco profundo, com presença de cascalho, baixa permeabilidade no subsolo, topografia ondulada e condições razoáveis de infiltração, e (d) $10 \%$ da área são cobertos por mata ciliar sobre solo bem estruturado, argiloso, de subsolo permeável e pouca declividade, favorecendo a infiltração.

Os pontos P1, P2, P3 e P4, apresentados na Figura 2, representam os locais de amostragem de solo para a determinação da umidade ao longo do ano, na microbacia de estudo. Os três pluviógrafos utilizados neste trabalho estavam distribuídos da seguinte forma: um na estação climatológica, um no ponto P2 e outro no ponto P4.

\section{Simulação da evapotranspiração}

Para a realização desta pesquisa, utilizou-se o método de Penman-FAO para a simulação da evapotranspiração de 


\section{A. Embrapa (1978)}

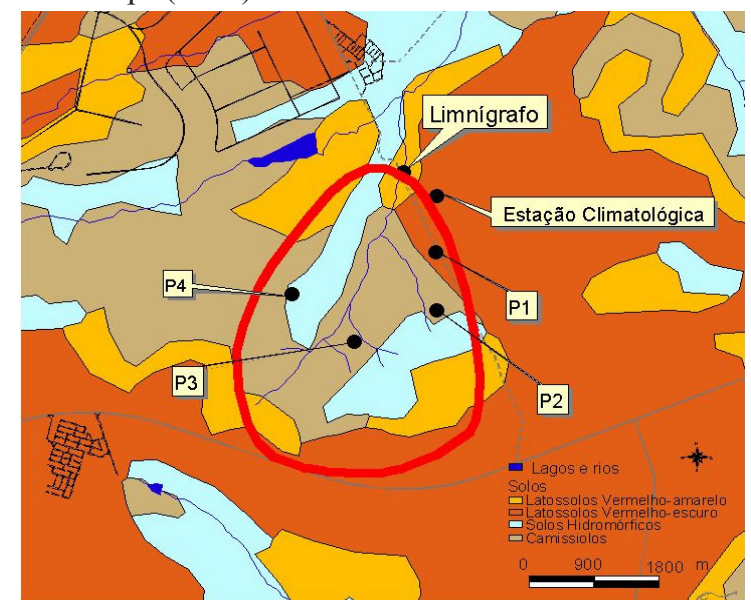

B. CODEPLAN (1994)

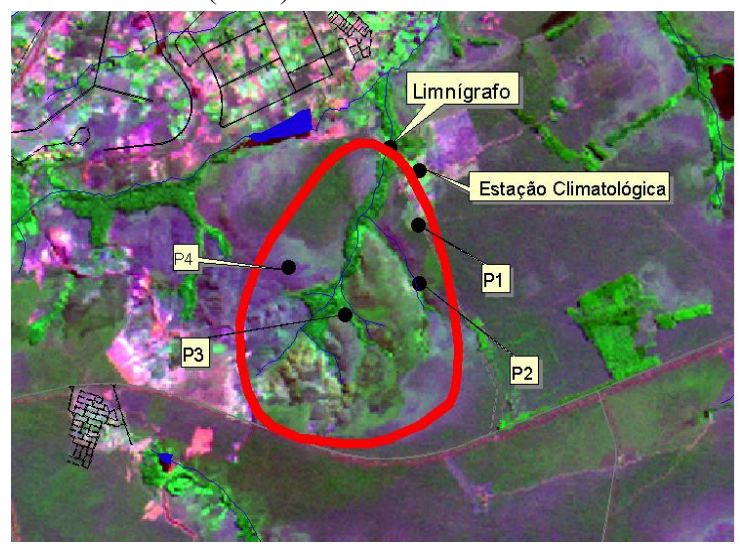

Figura 2. Mapas de caracterização física da microbacia do córrego do Capetinga (A) EMBRAPA (1978) e (B) CODEPLAN (1994)

referência (ETo) o qual constitui uma modificação do método original apresentado por Penman em 1948 (Doorenbos \& Pruitt, 1977).

Segundo Doorenbos \& Pruitt (1977) a equação de PenmanFAO pode ser descrita por:

$$
\mathrm{ETo}=\mathrm{c} \cdot[\mathrm{W} \cdot \mathrm{Rn}+(1-\mathrm{W}) \cdot \mathrm{f}(\mathrm{u}) \cdot(\mathrm{es}-\mathrm{ea})]
$$

em que:

ETo - evapotranspiração de referência, $\mathrm{mm} \mathrm{d}^{-1}$

c - fator regional de ajuste da equação

$\mathrm{W}$ - fator de ponderação relacionado à temperatura

$\mathrm{Rn}$ - saldo de radiação, equivalente em mm de água evaporada

$\mathrm{f}(\mathrm{u})$ - função relacionada ao vento

es - pressão de saturação do vapor da água, mbar

ea - pressão parcial de vapor da água, mbar

Segundo Cuenca (1989) o método de interpolação linear múltipla e a extrapolação podem ser utilizados para a determinação do fator "c" da tabela apresentada por Doorenbos \& Pruitt (1977).

Para facilitar a utilização de programas computacionais no cálculo do coeficiente de calibração (c) e da evapotranspiração pelo método de Penman-FAO, elaborou a seguinte equação (Cuenca, 1989), para a determinação deste fator:

$$
\begin{aligned}
\mathrm{c} & =0,68+0,0028\left(\mathrm{UR}_{\max }\right)+0,018(\mathrm{Rs})-0,068\left(\mathrm{U}_{2 \text { dia }}\right)+ \\
& +0,013\left(\frac{\mathrm{U}_{\mathrm{d}}}{\mathrm{U}_{\mathrm{n}}}\right)+0,0097\left(\mathrm{U}_{2 \text { dia }}\left(\frac{\mathrm{U}_{\mathrm{d}}}{\mathrm{U}_{\mathrm{n}}}\right)+0,43 \times 10^{-4}\left(\mathrm{UR}_{\max }\right)(\mathrm{Rs})\left(\mathrm{U}_{2 \text { dia }}\right)\right.
\end{aligned}
$$

em que:

$$
\begin{aligned}
& \mathrm{UR}_{\max } \text { - umidade relativa máxima do ar, porcentagem } \\
& \text { Rs - radiação solar global, } \mathrm{mm} \mathrm{d}^{-1} \\
& \mathrm{U}_{\text {2dia }} \text { - velocidade média do vento durante o dia, a } 2 \mathrm{~m} \text { de }
\end{aligned}
$$

Para a simulação da evapotranspiração efetuada neste trabalho, foi utilizada a Eq. 2 para a determinação do fator de ajuste c, como proposto por Cuenca \& Jensen (1988) e citado por Cuenca (1989).

A relação entre a evapotranspiração real da cultura ou vegetação (ETr) e a evapotranspiração de referência (ETo) de determinada área, pode ser representada pela seguinte equação (Bernardo, 1995):

$$
\mathrm{ETr}=\mathrm{ETo} . \mathrm{Kc} . \mathrm{Ks}
$$

em que:

Kc - coeficiente da vegetação ou da cultura

Ks - coeficiente que depende da umidade do solo

Os valores do coeficiente da cultura (Kc) são normalmente conhecidos para culturas irrigadas; entretanto, para vegetação natural, onde as plantas se encontram plenamente desenvolvidas, isto é, em perfeito equilíbrio entre o crescimento e a senescência, não faz sentido a adoção de um Kc variável; por tal razão, adotou-se o valor unitário para este parâmetro.

A medida em que a umidade do solo decresce, o fator $\mathrm{Ks}$ diminui, reduzindo a evapotranspiração real da cultura (ETr). Várias teorias e métodos foram estabelecidos para relacionar a disponibilidade de água no solo com o fator Ks. Na Figura 3 são apresentadas propostas de correlação entre esses parâmetros, desde as mais antigas (Thornthwaite \& Matter, $1955)$ até as mais modernas, como a curva Pierce proposta por Bernardo (1995).

Neste trabalho, foram efetuadas simulações da evapotranspiração real utilizando-se, para o cálculo de Ks, os métodos propostos por Thornthwaite \& Mather (1955) e

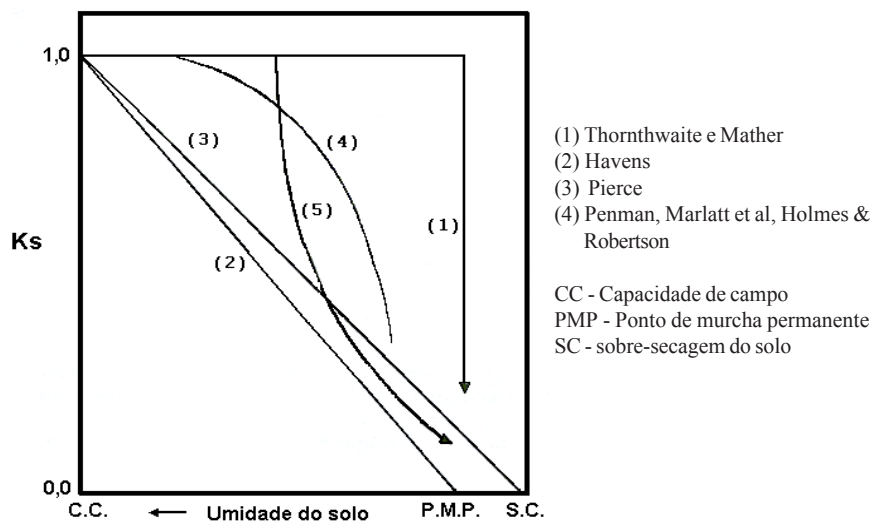

Figura 3. Propostas de relação do Ks com a umidade do solo 
Bernardo (1995). A razão desta escolha foi fundamentada pela lógica dos métodos. O primeiro considera que a evapotranspiração atual varia de um máximo igual a ETo, na condição de umidade correspondente à capacidade de campo, até um valor nulo, quando o solo alcança o ponto de murcha permanente. O proposto por Bernardo (1995) considera que o parâmetro Ks tem um valor máximo unitário correspondente à capacidade de campo, com uma queda em proporções logarítmicas entre os limites capacidade de campo e ponto de murcha permanente.

A lógica dos métodos é descrita pelas Eqs. 4 e 5 para os modelos de Thornthwaite \& Mather (1955) e Bernardo (1995), respectivamente.

Segundo dados apresentados por Kato (1995) a microbacia do córrego do Capetinga apresenta, em média, valores referentes à capacidade de campo (limite superior de água no solo) de $380 \mathrm{~mm} \mathrm{~m}^{-1}$ de solo e ao ponto de murcha permanente (limite inferior de água no solo) de $223 \mathrm{~mm} \mathrm{~m}^{-1}$ de solo.

Utilizando-se os dados apresentados pelo pesquisador supracitado e se considerando uma linha reta para o método de Thonthwaite \& Mather (1955) chegou-se à seguinte equação, para o parâmetro Ks.

$$
\mathrm{Ks}=0,00637(\mathrm{LTA})-1,4204
$$

em que:

LTA - lâmina total de água armazenada no solo em mm

Para o método de Bernardo (1995) o parâmetro Ks é dado por:

$$
\mathrm{Ks}=\frac{\operatorname{Ln}((\mathrm{UA}-\mathrm{PM})+1)}{\operatorname{Ln}((\mathrm{CC}-\mathrm{PM})+1)}=\frac{\operatorname{Ln}(\mathrm{LAA}+1)}{\operatorname{Ln}(\mathrm{CTA}+1)}
$$

em que:

CC - teor de umidade do solo na capacidade de campo em mm de água

PM - teor de umidade do solo no ponto de murchamento em mm de água

UA - teor de umidade atual do solo em mm de água

LAA - lâmina atual de água no solo em $\mathrm{mm}$ de água

CTA - capacidade total de água no solo em mm de água

Para a elaboração das simulações realizadas utilizaram-se os princípios do balanço hídrico, da seguinte forma:

$$
\mathrm{LTA}_{(\mathrm{i})}=\mathrm{LTA}_{(\mathrm{i}-1)}+\mathrm{P}_{(\mathrm{i}-1)}-\mathrm{ETr}_{(\mathrm{i}-1)}
$$

em que:

LTA $_{(i)}$ - lâmina total atual de água no solo no dia i, mm

$\mathrm{LTA}_{(\mathrm{i}-1)}$ - lâmina total de água no solo do dia anterior ao dia $\mathrm{i}, \mathrm{mm}$

$\mathrm{P}_{(\mathrm{i}-1)} \quad$ - precipitação do dia anterior ao dia i, mm

$\mathrm{ETr}_{(\mathrm{i}-1)}$ - evapotranspiração real do dia anterior ao dia i, mm

Na simulação, todas as vezes em que a lâmina total atual de água no solo (LTA) apresentou valores acima do limite superior de água no solo, $380 \mathrm{~mm} \mathrm{~m}^{-1}$ de solo, a lâmina excedente foi computada como escoamento total.
O valor correspondente à lâmina total de água armazenada no solo no início da simulação, foi obtido por Lima (2000) na área em estudo.

$\mathrm{Na}$ atualidade, com os avanços das técnicas computacionais, tornou-se menos trabalhosa a solução do modelo clássico de Richards na simulação da água infiltrada no solo e da redistribuição da mesma ao longo de seu perfil. A grande dificuldade da utilização do modelo de Richards está na sua alimentação com parâmetros físico-hídricos do solo, representativos de grandes áreas, devido à variabilidade espacial, principalmente quanto à curva característica de umidade e à condutividade hidráulica. razão pela qual se adotou um modelo simples para a simulação da lâmina total de água no solo, em detrimento de um modelo fisicamente perfeito.

Os dados de variação da velocidade do vento durante o dia $\left(\mathrm{U}_{\text {dia }}\right)$ e durante a noite $\left(\mathrm{U}_{\text {noite }}\right)$ foram obtidos no Instituto Nacional de Meteorologia - INMET, o mesmo ocorrendo com os dados de insolação (n). A estação em que foram tomados esses dados fica dentro do próprio INMET, a aproximadamente $15 \mathrm{~km}$, em linha reta, da microbacia do córrego do Capetinga.

A literatura especializada recomenda a utilização do método Penman-Monteith na simulação da evapotranspiração de referência; porém, nas condições experimentais do presente trabalho não se dispunha de saldo radiômetro; portanto, a radiação líquida foi calculada seguindo-se as recomendações da FAO, razão por que se adotou o método Penman-FAO para a determinação de Eto, utilizando-se dados da estação experimental da Fazenda Água Limpa, próximo aos limites da bacia estudada.

\section{RESULTADOS E DISCUSSÃO}

\section{Determinação da evapotranspiração de referência (ETo) pelo método Penman-FAO}

A Tabela 1 apresenta os dados utilizados para a aplicação do método Penman-FAO e os resultados obtidos para os intervalos de tempo estudados. É importante ressaltar que os valores de ETo foram determinados diariamente. Posteriormente, agruparam-se os resultados em períodos estabelecidos conforme as necessidades do trabalho de Lima (2000).

Na Figura 4 tem-se os valores da evapotranspiração de referência (ETo) obtidos através do método Penman-FAO. Os pontos do gráfico abaixo representam os valores médios dos períodos analisados e obtidos através da Eq. 1, enquanto o traço contínuo representa a linha de tendência dos mesmos ao longo do ano.

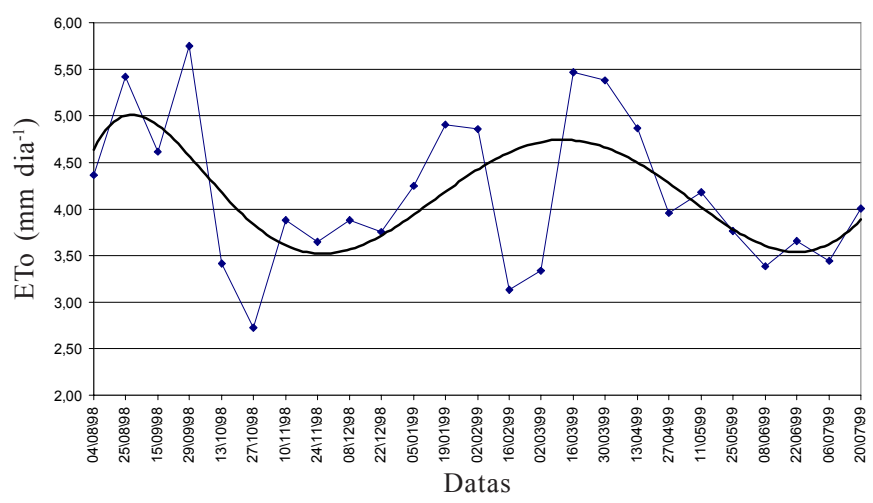

Figura 4. Representação gráfica da evapotranspiração de referência calculada pelo método Penman-FAO para a microbacia do córrego do Capetinga 
Tabela 1. Demonstrativo de cálculo da evapotranspiração de referência (ETo) pelo método Penman-FAO

\begin{tabular}{|c|c|c|c|c|c|c|c|c|c|c|c|c|c|c|c|c|c|c|}
\hline \multirow{2}{*}{ Período } & méd & es & ea & $\mathrm{R}_{\text {méd }}$ & $\mathrm{UR}_{\text {má }}$ & \multirow{2}{*}{$\begin{array}{c}\mathrm{U} 2 \\
\mathrm{~km} \mathrm{~d}^{-1}\end{array}$} & \multirow[t]{2}{*}{$f(u)$} & \multirow[t]{2}{*}{$f(t)$} & \multirow[t]{2}{*}{$f(e a)$} & \multirow[t]{2}{*}{$f(n / N)$} & \multirow[t]{2}{*}{$\mathrm{w}$} & & Rs & & & $\mathrm{Rn}$ & & \multirow[t]{2}{*}{ fator $\mathrm{c}$} \\
\hline & ${ }^{\circ} \mathrm{C}$ & \multicolumn{2}{|c|}{ mbar } & \multicolumn{2}{|c|}{$\%$} & & & & & & & \multicolumn{6}{|c|}{$\mathrm{mm} \mathrm{d}^{-1}$} & \\
\hline $04 / 08 / 98$ & 23,33 & 3,66 & 120 & & 54,0 & 101,84 & & 15,21 & & & & & & & 2,44 & & & \\
\hline & 4,05 & & & & & & & & & & & & & & & & & \\
\hline & & & & & & & & & & & & & & & & & & \\
\hline & & & & & & & & & & & & & & & & & & \\
\hline & & & & & & & & & & & & & & & & & & \\
\hline &, 58 & & & & & & & & & & & & & & & & & \\
\hline & & & & & & & & & & & & & & & & & & \\
\hline & & & & & & & & & & & & & & & & & & \\
\hline & & & & & & & & & & & & & & & & & & \\
\hline & & & & & & & & & & & & & & & & & & \\
\hline & & & & & & & & & & & & & & & & & & \\
\hline & & & & & & & & & & & & & & & & & & \\
\hline & 2304 & & & & & & & & & & & & & & & & & \\
\hline & 21,53 & & & & & & & & & & & 3,00 & & 4,5 & 1,63 & 2,93 & 3,14 & \\
\hline & 3,05 & & & & & & & & & & & & & 4,6 & 1,4 & 3 , & & \\
\hline & 25,06 & & & & & & & & & & & & & & 1,4 & 5,36 & 5,46 & 924 \\
\hline & 27,04 & & & & & & & & & & & & & & 1,8 & 4,97 & 5,38 & 323 \\
\hline & 24,56 & & & & & & & & & & & & & & 1,9 & 4,57 & 4,86 & 576 \\
\hline & 2020 & & & & & & & & & & & & & 5,7 & 2,23 & 3,50 & 3,95 & 0,9573 \\
\hline & & & & & & & & & & & & & & & 2,4 & 3,67 & & \\
\hline & & & & & & & & & & & & & & & 2,4 & & & \\
\hline & & & & & & & & & & & & & & & 2,2 & & & \\
\hline & & & & & & & & & & & & & & & 2,4 & & & \\
\hline & & & & & & & & & & & & & & & & & & \\
\hline & & & & & & & & & & & & & & & & & & \\
\hline 04/08/99 & 22,91 & & & & & & & & & & & & & 5,61 & 1,79 & & & דיד, \\
\hline
\end{tabular}

Simulação da evapotranspiração real da bacia, a partir dos dados de evapotranspiração de referência, obtidos pelo método Penman-FAO

A Tabela 2 apresenta um exemplo da simulação com a utilização do fator Ks, obtido segundo a Eq. 4 (Bernardo, 1995). A capacidade total de armazenamento de água no solo (CTA) é igual ao seu limite superior, apresentado anteriormente como sendo, para a área estudada, de $380 \mathrm{~mm} \mathrm{~m}^{-1}$ de solo. Como

Tabela 2. Exemplo da simulação da lâmina total armazenada no solo (LTA), do escoamento e da evapotranspiração real (ETr) utilizando-se o fator Ks proposto por Bernardo (1995)

\begin{tabular}{|c|c|c|c|c|c|c|}
\hline \multirow{2}{*}{ Data } & \multirow{2}{*}{$\begin{array}{c}\text { LTA } \\
\mathrm{mm} \mathrm{m}^{-1}\end{array}$} & \multirow{2}{*}{$\begin{array}{l}\text { ETo } \\
\mathrm{mm}\end{array}$} & \multirow[t]{2}{*}{ Ks } & ETr & Precipitação & Escoamento \\
\hline & & & & \multicolumn{3}{|c|}{$\mathrm{mm}$} \\
\hline $04 / 08 / 98$ & 237,60 & 3,94 & 0,47 & 1,70 & 3,10 & 0,00 \\
\hline $25 / 08 / 98$ & 223,00 & 4,84 & 0,00 & 0,00 & 0,00 & 0,00 \\
\hline $15 / 09 / 98$ & 223,00 & 4,49 & 0,00 & 0,00 & 0,00 & 0,00 \\
\hline 29/09/98 & 226,10 & 4,55 & 0,23 & 1,04 & 41,83 & 0,00 \\
\hline $13 / 10 / 98$ & 271,54 & 2,18 & 0,76 & 1,64 & 53,20 & 0,00 \\
\hline $27 / 10 / 98$ & 320,94 & 1,98 & 0,90 & 1,78 & 88,78 & 0,00 \\
\hline $10 / 11 / 98$ & 373,28 & 2,82 & 0,99 & 2,80 & 162,45 & 91,32 \\
\hline $24 / 11 / 98$ & 378,52 & 2,44 & 1,00 & 2,43 & 119,97 & 85,48 \\
\hline 08/12/98 & 379,45 & 2,45 & 1,00 & 2,45 & 90,67 & 56,44 \\
\hline $22 / 12 / 98$ & 376,91 & 2,62 & 1,00 & 2,61 & 69,98 & 31,87 \\
\hline 05/01/99 & 369,22 & 3,06 & 0,99 & 3,03 & 38,04 & 0,00 \\
\hline 19/01/99 & 353,38 & 3,93 & 0,96 & 3,79 & 30,50 & 0,00 \\
\hline $02 / 02 / 99$ & 342,11 & 3,51 & 0,95 & 3,31 & 31,52 & 0,00 \\
\hline $16 / 02 / 99$ & 357,39 & 2,56 & 0,97 & 2,47 & 107,39 & 0,00 \\
\hline 02/03/99 & 378,75 & 2,24 & 1,00 & 2,24 & 135,35 & 118,16 \\
\hline $16 / 03 / 99$ & 364,04 & 3,82 & 0,98 & 3,72 & 23,90 & 31,51 \\
\hline 30/03/99 & 311,69 & 4,41 & 0,89 & 3,91 & 8,96 & 0,00 \\
\hline $13 / 04 / 99$ & 291,97 & 3,96 & 0,84 & 3,32 & 36,67 & 0,00 \\
\hline $27 / 04 / 99$ & 264,72 & 3,45 & 0,74 & 2,56 & 14,92 & 0,00 \\
\hline $11 / 05 / 99$ & 246,57 & 3,17 & 0,62 & 1,94 & 0,00 & 0,00 \\
\hline $25 / 05 / 99$ & 225,62 & 3,28 & 0,20 & 0,72 & 1,50 & 0,00 \\
\hline 08/06/99 & 223,04 & 3,00 & 0,01 & 0,02 & 0,00 & 0,00 \\
\hline $22 / 06 / 99$ & 223,00 & 3,31 & 0,00 & 0,00 & 0,00 & 0,00 \\
\hline $06 / 07 / 99$ & 223,00 & 3,30 & 0,00 & 0,00 & 0,00 & 0,00 \\
\hline \multirow[t]{2}{*}{$20 / 07 / 99$} & 223,00 & 3,87 & 0,00 & 0,00 & 0,00 & 0,00 \\
\hline & $-32,68^{*}$ & 1217,88 & & 676,64 & 1058,73 & 414,78 \\
\hline
\end{tabular}

* O valor indicado representa a diferença entre a LTA do dia 04/08/98 e do dia 03/08/99; $\mathrm{Kc}=1$ 
citado antes, o primeiro valor de LTA para o dia 4 de agosto de 1998, foi medido e apresentado por Lima (2000) e os valores seguintes foram simulados a partir da Eq. 6. Os valores de evapotranspiração de referência (ETo) foram calculados segundo o método Penman-FAO, apresentado na Eq. 1. O valor de $\mathrm{Kc}$ foi considerado constante e igual a 1 . O valor da evapotranspiração real foi determinado a partir da Eq. 3. A precipitação foi medida e apresentada por Lima (2000). O escoamento, conforme explicado anteriormente, foi determinado pela diferença entre os valores de LTA e CTA, todas as vezes em que LTA excedeu a CTA.

$O$ resultado final obtido pela simulação exemplificada na Tabela 2 foi que, no intervalo de um ano analisado, a lâmina de água armazenada no solo reduziu em $32,68 \mathrm{~mm} \mathrm{~m}^{-1}$ de solo, a evapotranspiração real total foi de $676,64 \mathrm{~mm}$ e o escoamento total foi de $414,78 \mathrm{~mm}$.

A Tabela 3 seguiu os mesmos procedimentos de cálculo apresentados na Tabela 2, porém os valores de Ks foram determinados pelo método proposto por Thornthwaite \& Mather (1955) apresentados na Eq. 5.

$\mathrm{Na}$ simulação apresentada na Tabela 3, os valores encontrados apresentaram redução na lâmina total de água armazenada no solo, de $17,93 \mathrm{~mm} \mathrm{~m}^{-1}$ de solo, a evapotranspiração real total foi de $616,43 \mathrm{~mm}$ e o escoamento total foi de $460 \mathrm{~mm}$

A Figura 5 apresenta os valores de evapotranspiração real obtidos experimentalmente por Lima (2000), os valores simulados neste trabalho e os valores da evapotranspiração de referência, determinados pelo método Penman-FAO.

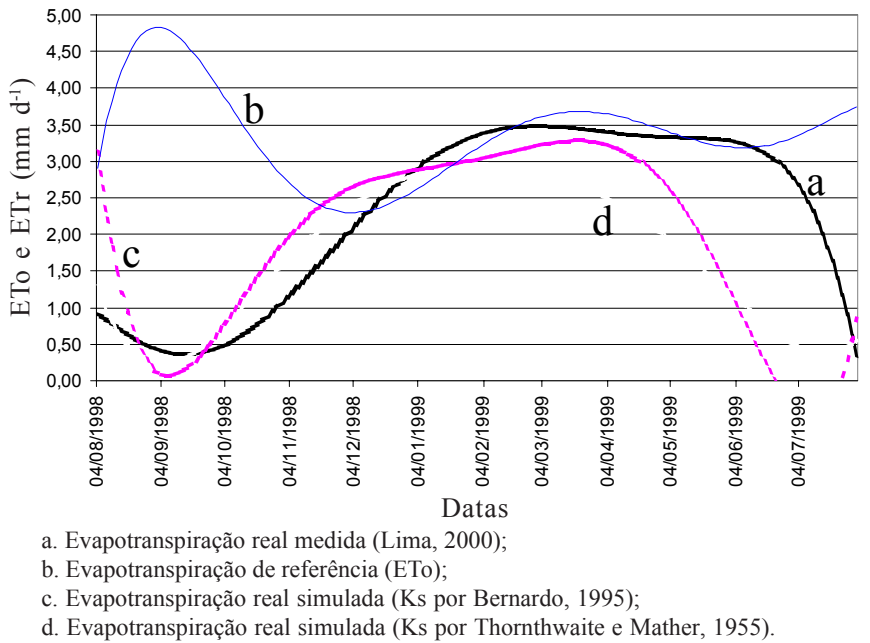

Figura 5. Gráfico comparativo entre a evapotranspiração real simulada utilizando-se dois métodos de determinação do Ks, a evapotranspiração de referência calculada pelo método de Penman-FAO e a evapotranspiração real medida por Lima (2000) para a microbacia do córrego Capetinga, para o período hidrológico 1998/1999

Pode-se observar, na Figura 5, que os valores simulados de evapotranspiração real durante o período compreendido entre os meses de julho e dezembro, apresentaram comportamento similar aos valores obtidos por Lima (2000); entretanto, no restante do período analisado constata-se uma subestimativa da evapotranspiração real simulada, pelos dois métodos utilizados. De julho até dezembro, como é demonstrado na Figura

Tabela 3. Exemplo da simulação da lâmina total armazenada no solo (LTA), do escoamento total e da evapotranspiração real (ETr) utilizando-se o fator Ks proposto por Thornthwaite \& Mather (1955)

\begin{tabular}{|c|c|c|c|c|c|c|}
\hline \multirow{2}{*}{ Data } & LTA & \multirow{2}{*}{$\begin{array}{l}\text { ETo } \\
\text { mm }\end{array}$} & \multirow[t]{2}{*}{$\mathrm{Ks}$} & $\mathrm{ETr}$ & Precipitação & Escoamento \\
\hline & $\mathrm{mm} \mathrm{m}^{-1}$ & & & \multicolumn{3}{|c|}{$\mathrm{mm}$} \\
\hline $04 / 08 / 98$ & 251,05 & 3,94 & 0,18 & 0,69 & 3,10 & 0,00 \\
\hline $25 / 08 / 98$ & 238,88 & 4,84 & 0,10 & 0,49 & 0,00 & 0,00 \\
\hline $15 / 09 / 98$ & 231,95 & 4,49 & 0,06 & 0,26 & 0,00 & 0,00 \\
\hline $29 / 09 / 98$ & 237,80 & 4,55 & 0,09 & 0,42 & 41,83 & 0,00 \\
\hline $13 / 10 / 98$ & 292,51 & 2,18 & 0,44 & 0,95 & 53,20 & 0,00 \\
\hline $27 / 10 / 98$ & 348,75 & 1,98 & 0,80 & 1,56 & 88,78 & 0,00 \\
\hline $10 / 11 / 98$ & 378,43 & 2,82 & 0,99 & 2,79 & 162,45 & 91,32 \\
\hline $24 / 11 / 98$ & 378,55 & 2,44 & 0,99 & 2,41 & 119,97 & 85,48 \\
\hline $08 / 12 / 98$ & 379,46 & 2,45 & 1,00 & 2,44 & 90,67 & 56,44 \\
\hline $22 / 12 / 98$ & 376,96 & 2,62 & 0,98 & 2,57 & 69,98 & 31,87 \\
\hline $05 / 01 / 99$ & 370,10 & 3,06 & 0,94 & 2,91 & 38,04 & 0,00 \\
\hline $19 / 01 / 99$ & 356,62 & 3,93 & 0,85 & 3,38 & 30,50 & 0,00 \\
\hline $02 / 02 / 99$ & 351,78 & 3,51 & 0,82 & 2,87 & 31,52 & 0,00 \\
\hline $16 / 02 / 99$ & 367,83 & 2,56 & 0,92 & 2,32 & 107,39 & 0,00 \\
\hline $02 / 03 / 99$ & 378,77 & 2,24 & 0,99 & 2,22 & 135,35 & 118,16 \\
\hline $16 / 03 / 99$ & 365,05 & 3,82 & 0,90 & 3,41 & 23,90 & 31,51 \\
\hline $30 / 03 / 99$ & 322,91 & 4,41 & 0,64 & 2,82 & 8,96 & 0,00 \\
\hline $13 / 04 / 99$ & 318,08 & 3,96 & 0,61 & 2,40 & 36,67 & 0,00 \\
\hline 27/04/99 & 303,05 & 3,45 & 0,51 & 1,76 & 14,92 & 0,00 \\
\hline $11 / 05 / 99$ & 293,88 & 3,17 & 0,45 & 1,41 & 0,00 & 0,00 \\
\hline $25 / 05 / 99$ & 275,32 & 3,28 & 0,33 & 1,10 & 1,50 & 0,00 \\
\hline 08/06/99 & 263,68 & 3,00 & 0,26 & 0,78 & 0,00 & 0,00 \\
\hline $22 / 06 / 99$ & 253,56 & 3,31 & 0,19 & 0,65 & 0,00 & 0,00 \\
\hline $06 / 07 / 99$ & 245,74 & 3,30 & 0,14 & 0,48 & 0,00 & 0,00 \\
\hline \multirow[t]{2}{*}{$20 / 07 / 99$} & 240,05 & 3,87 & 0,11 & 0,42 & 0,00 & 0,00 \\
\hline & $-17,93^{*}$ & 1217,88 & & 616,43 & 1058,73 & 460,64 \\
\hline
\end{tabular}


5, a evapotranspiração real e a de referência têm comportamento inversamente proporcional, enquanto no período de janeiro a junho a evapotranspiração real medida tem comportamento e valores próximos aos da evapotranspiração potencial determinada pelo método Penman-FAO.

A Figura 6 apresenta comparações efetuadas entre os valores de evapotranspiração de referência e real obtidas neste trabalho e nas medições efetuadas por Lima (2000). Foram feitas comparações entre a evapotranspiração real medida e a evapotranspiração de referência, entre as duas simulações realizadas neste trabalho, entre a simulação utilizando-se Ks dado por Bernardo (1995) e os valores medidos por Lima (2000) e entre a simulação utilizando-se Ks proposto por Thornthwaite \& Mather (1955) e os valores medidos por Lima (2000) representadas na Figura 6, como a, b, c e d, respectivamente.

Através da Figura 6A é possível observar-se que não houve correlação entre a evapotranspiração de referência (ETo) e a evapotranspiração real (ETr). Observando-se a Figura 5, evidencia-se que o comportamento da evapotranspiração real é o inverso da evapotranspiração de referência no período compreendido entre os meses de julho a agosto, conforme supracitado; isto ocorre devido ao fato de, neste período, a

A.

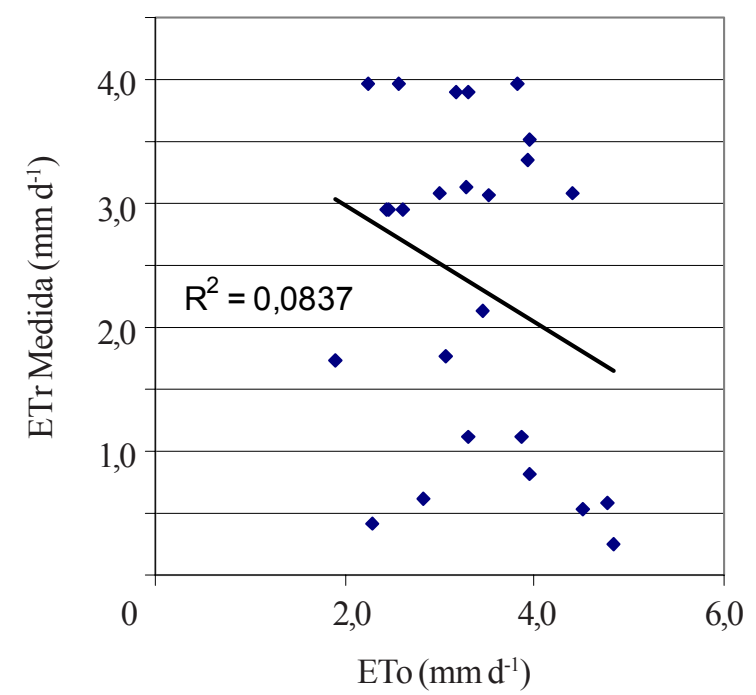

C.

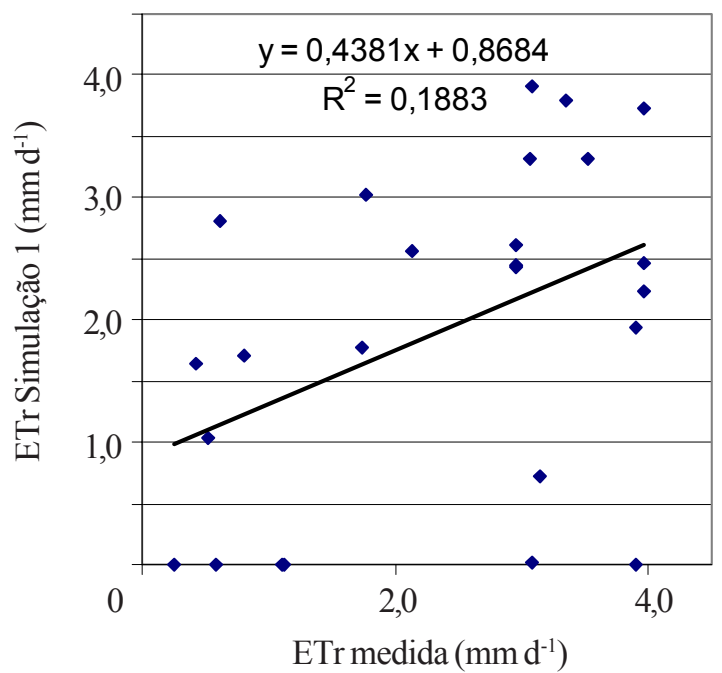

demanda atmosférica é muito alta; no entanto, não existe no solo água disponível para evapotranspirar.

Pela Figura 6B, observa-se que houve alta correlação entre os valores simulados de evapotranspiração real encontrados neste trabalho, mostrando que a variação de Ks utilizando-se os métodos de Thornthwaite \& Mather (1955) e de Bernardo (1995) são semelhantes, não obstante a época em que foram apresentados. Por outro lado, pelas Figuras 6C e 6D verificou-se baixa correlação entre os valores simulados e os medidos por Lima (2000) mostrando que as simulações não foram satisfatórias na determinação de ETr.

A Figura 7 apresenta os valores da variação da lâmina total de água armazenada no solo até um metro de profundidade, medidos por Lima (2000) ao longo do período hidrológico analisado e os simulados neste trabalho.

Observa-se, na Figura 7, que a simulação da lâmina de água presente no solo ao longo do ano apresentou valores similares aos medidos por Lima (2000).

Na Figura 8 são comparados: a) a lâmina de água no solo até um metro de profundidade, medida por Lima (2000) e a simulada utilizando Ks por Bernardo (1995); b) a lâmina de água no solo até um metro de profundidade medida por Lima (2000)

B.

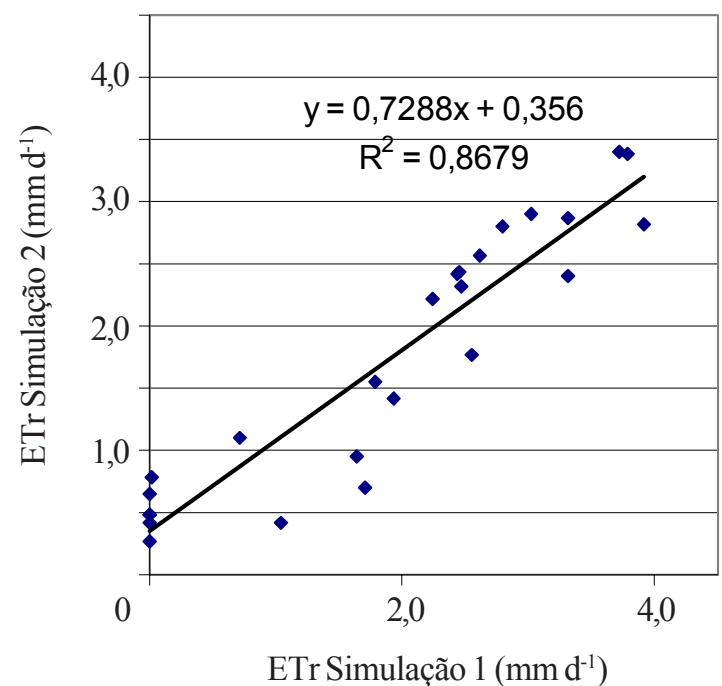

D.

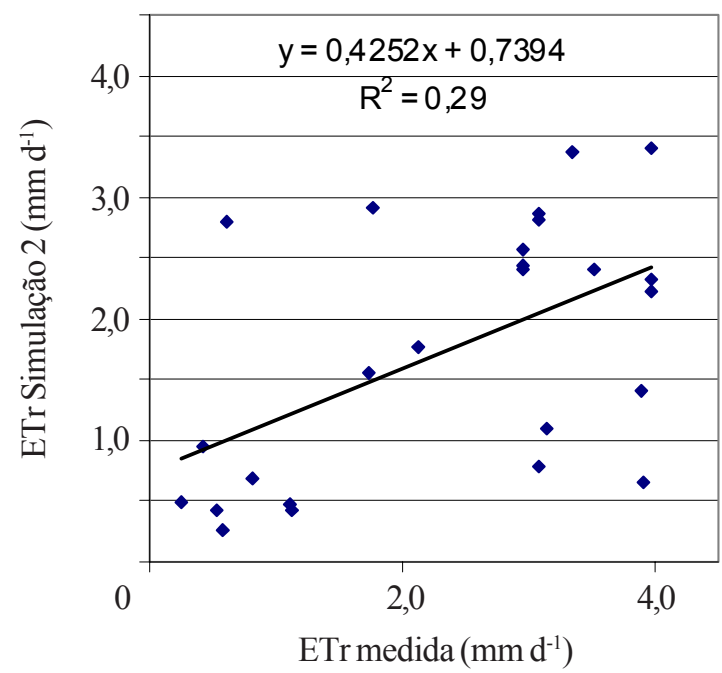

Figura 6. Análise de correlação entre os dados de evapotranspiração apresentados 


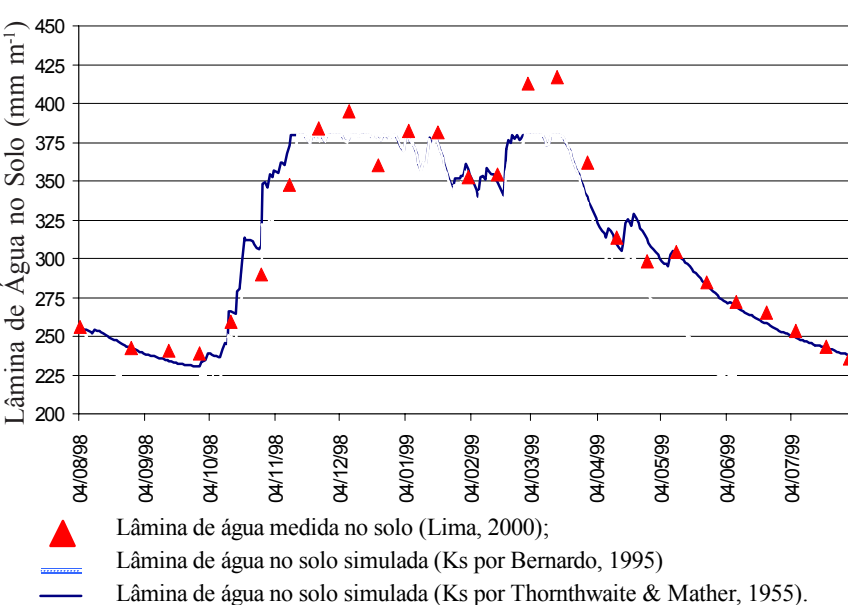

Figura 7. Gráfico comparativo entre as lâminas de água no solo simuladas, com os valores medidos por Lima (2000) até um metro de profundidade, para a microbacia do córrego Capetinga para o período hidrológico 1998/1999

e a simulada utilizando Ks por Thornthwaite \& Mather (1955) e c) as duas simulações.

Nas Figuras 7 e 8 é possível observar-se que os dois métodos de simulação utilizados apresentaram alta correlação $\left(\mathrm{R}^{2}=0,9529\right)$ quanto à variação da lâmina de água presente no solo, ao longo do ano. Além disso, ambos apresentaram alta correlação com os valores medidos por Lima (2000).

Na Tabela 4 são demonstrados os resultados totais obtidos no período hidrológico analisado através das medições efetuadas por Lima (2000) e pelas simulações efetuadas neste trabalho.

Conforme explicitado na Tabela 4, os valores de evapotranspiração real simulados ao longo do ano, pelos dois métodos descritos neste trabalho, apresentaram subestimativa em relação aos valores medidos por Lima (2000), confirmando os resultados citados anteriormente.

Pode-se observar, na Tabela 4 que, além da subestimativa da evapotranspiração real, as simulações apresentaram valores superestimados de escoamento ao longo do ano analisado. Certamente este fato deveria ocorrer, visto que, como apresentado nas Figuras 7 e 8, a simulação da lâmina de água armazenada no solo ao longo do ano apresentou alta correlação com os valores medidos.

O valor de $71,77 \mathrm{~mm}$ de variação da lâmina de água armazenada no solo ao longo do ano medido por Lima (2000) corresponde às zonas saturada e não saturada do solo enquanto os demais valores resultantes das simulações só consideraram a variação até um metro de profundidade do solo. Lima (2000) observou que a redução da lâmina de água armazenada no solo ao longo do ano analisado na zona não saturada foi de $24,3 \mathrm{~mm}$, valor este muito próximo aos obtidos nas simulações.

$\mathrm{O}$ valor relativo ao coeficiente de cultura ou vegetação $(\mathrm{Kc})$ utilizado neste trabalho foi considerado como sendo constante durante todo o ano, o que pode ter contribuído como uma das principais fontes de erro para as simulações elaboradas. Para solução deste viés, buscou-se uma tentativa para a determinação do Kc, visto que, de posse dos dados da evapotranspiração de referência (ETo), da evapotranspiração real medida (ETr) e do coeficiente relativo à umidade do solo $(\mathrm{Ks})$ através da Eq. 3, este poderia ser determinado, porém, como pode ser observado na Figura 3, existem vários métodos diferentes para a determinação do Ks e, como se nota nos exemplos de simulação (Tabelas 2 e 3), para uma mesma lâmina de água no solo $\left(255,68 \mathrm{~mm} \mathrm{~m}^{-1}\right)$, quando utilizada a metodologia de Bernardo (1995) o valor de Ks é igual a 0,6947 e, quando utilizada a metodologia de Thornthwaite \& Mather(1955) Ks é 0,2083.
A.

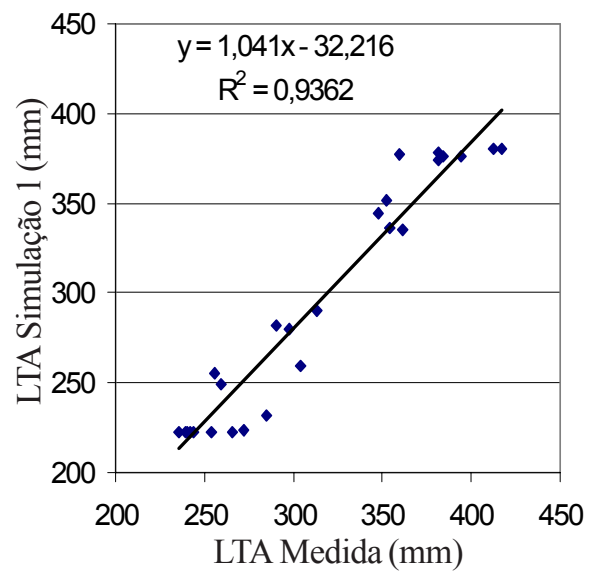

B.

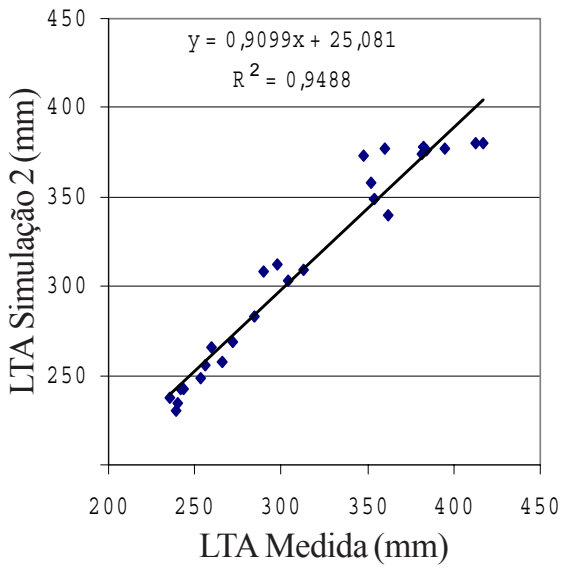

C.

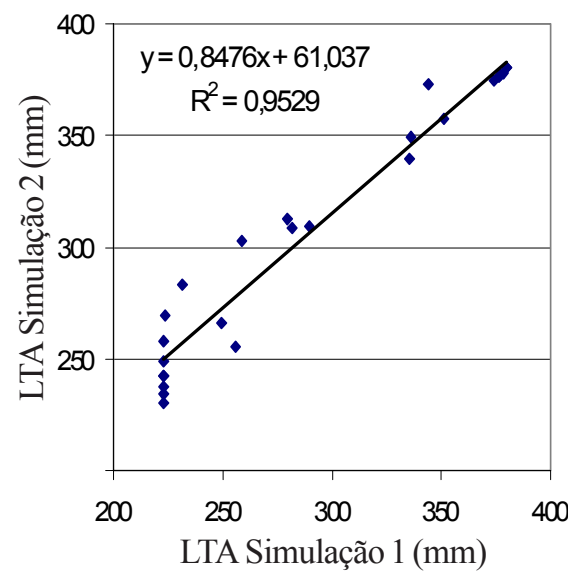

Figura 8. Análise de correlação entre as lâminas da água armazenada no solo (LTA), medidas por Lima (2000) e os valores encontrados nas duas simulações realizadas neste trabalho, ao longo do período hidrológico 1998/1999

Tabela 4. Resumo dos resultados obtidos

\begin{tabular}{lccc}
\multicolumn{1}{c}{ Parâmetro } & $\begin{array}{c}\text { Medido } \\
\text { (Lima, 2000) }\end{array}$ & $\begin{array}{c}\text { Simulação 1 } \\
\text { (Ks por Bernardo, 1995) }\end{array}$ & $\begin{array}{c}\text { Simulação 2 } \\
\text { (Ks por Thornthwaite \& Mather, 1955) }\end{array}$ \\
\hline Precipitação (P) - mm & 1058,73 & 1058,73 & 1058,73 \\
Evapotranspiração real (ETr) $-\mathrm{mm}$ & 831,03 & 676,64 & 616,43 \\
Escoamento total (Es + Eb) - mm & 299,47 & 414,78 & 460,64 \\
Variação da lâmina de água armazenada & $-71,77$ & $-32,68$ & $-17,93$ \\
$\quad$ no solo (dS/dt) - mm & & & \\
\hline
\end{tabular}


Mesmo se obtendo alta correlação entre as lâminas de água no solo (LTA) simuladas nesta pesquisa e os valores medidos por Lima (2000) antes de afirmar que as medições de campo deste parâmetro, durante um período hidrológico, possam ser substituídas por valores simulados segundo a metodologia descrita neste trabalho, é fundamental que, anteriormente, se conheça melhor o comportamento do coeficiente de vegetação (Kc) e do coeficiente relativo ao teor de umidade do solo (Ks) da área de estudo.

\section{CONCLUSÕES}

1. Os valores simulados de evapotranspiração real e escoamento total não apresentaram boa correlação com os dados obtidos experimentalmente por Lima (2000) para a mesma área e período hidrológico.

2. Os dois métodos de determinação do coeficiente de umidade do solo $(\mathrm{Ks})$ apresentados neste trabalho não acarretaram mudanças significativas nos resultados finais obtidos

3. Os valores simulados da lâmina total de água no solo (LTA) até um metro de profundidade, ao longo do ano, apresentaram-se muito próximos aos valores medidos por Lima (2000).

\section{LITERATURA CITADA}

Beekman, G.B. Gerenciamento integrado dos recursos hídricos. Brasília: IICA, 1999. 64p.

Berlato, M.A.; Molion, L.C.B. Evaporação e evapotranspiração. Boletim Técnico, v.7, Porto Alegre: IPAGRO, 1981. 95p.

Bernardo, S. Manual de irrigação. $6^{\mathrm{a}}$ ed. Viçosa: UFV, Imprensa Universitária, 1995. 657p.

Chang, J. Climate and agriculture: An ecological survey. Chicago, 1968. 304p.

CODEPLAN, IBGE. Projeção da população da região Centro Oeste e Tocantins. In: Jornal Correio Braziliense de 28 de janeiro de 2000, Caderno Cidade, 2000.
Cuenca, R.H. Irrigation system design: An engineereing approach. New Jersey: Prentice Hall. 1989. 552p.

Doorenbos, J.; Pruitt, W.O. Guidelines for predicting crop water requirements. FAO Irrigation and Drainage paper 24, Rome: FAO, 1977.

EMBRAPA. Levantamento de reconhecimento dos solos do Distrito Federal, n.53, Rio de Janeiro: SNLCS, 1978. 455 p. Boletim Técnico

Jensen, M.E. Consumptive use of water and irrigation water requirements. New York: American Society of Civil Engineers, 1973.215p.

Lima, J.E.F.W. Determinação da evapotranspiração de uma bacia hidrográfica sob vegetação natural de cerrado, pelo método do balanço hídrico. Departamento de Engenharia Agronômica, UnB, Brasília, 2000. Dissertação Mestrado

Mello, J.L.P.; Sediyama, G.C.; Pruski, F.F.; Silva, D.D. Evaporação e evapotranspiração. Viçosa: UFV. 1996. 62p.

Ortolani, A.A.; Camargo, A.P.; Nova, N.A.V. Correlação entre valores decendiais da evapotranspiração potencial calculados segundo métodos de Penman e de Thornthwaite e dados de evapotranspirômetros, na região de Ribeirão Preto. Bragantia, Campinas, p.26-41, 1966.

Rebouças, A.C.; Braga, B.; Tundizi, J.G. Águas doces no Brasil: capital ecológico, uso e conservação. Escrituras ed., São Paulo 1999. 717p.

Silva, C.L.; Oliveira, C.A.S. Runoff measurement and prediction for a watershed under natural vegetation in Central Brazil, Revista Brasileira de Ciência do Solo, Viçosa, v.23, n.3, p.695-701 1999.

Silva, M.A.V. Evapotranspiração em cultura irrigada no sub-médio São Francisco. São José dos Campos: INPE, 1977. 97p. Dissertação Mestrado

Silva, M.A.V. Meteorologia e climatologia. Brasília: INMET, 2000. $532 p$.

Thornthwaite, C.W.; Mather, J.R. The water balance. Climatology, Centerton, NJ. v.8, n.1. 1955. 